\title{
Critical Re-evaluation of the Slope Factor of the Operational Model of Agonism Short Title: Slope Factor of the Operational Model of Agonism
}

Jan Jakubík ( $\square$ jan.jakubik@fgu.cas.cz )

Czech Academy of Sciences

Alena Randáková

Czech Academy of Sciences

Dominik Nelic

Czech Academy of Sciences

\section{Research Article}

Keywords: Receptor theory, operational model of agonism, operational efficacy, slope factor

Posted Date: September 20th, 2021

DOI: https://doi.org/10.21203/rs.3.rs-900678/v1

License: (c) (i) This work is licensed under a Creative Commons Attribution 4.0 International License.

Read Full License 


\section{Critical re-evaluation of the slope factor of the operational model of agonism}

Short title: Slope factor of the operational model of agonism

Jan Jakubík, Alena Randáková, Dominik Nelic

Institute of Physiology Czech Academy of Sciences, Prague, Czech Republic

Correspondence: Jan Jakubik, Inst. of Physiology CAS, Vídeňská 1083, 14220 Praha, Czech

Republic, jan.jakubik@fgu.cas.cz 


\begin{abstract}
Although being a relative term, agonist efficacy is a cornerstone in the proper assessment of agonist selectivity and signalling bias. The operational model of agonism (OMA) has become successful in the determination of agonist efficacies and ranking them. In 1985, Black et al. introduced the slope factor to the OMA to make it more flexible and allow for fitting steep as well as flat concentration-response curves. Functional analysis of OMA demonstrates that the slope factor implemented by Black et al. affects relationships among parameters of the OMA. Fitting of the OMA with Black et al. slope factor to concentration-response curves of experimental as well as theoretical data (homotropic allosteric modulation, substrate inhibition and non-competitive auto-inhibition) resulted in wrong estimates of operational efficacy and affinity. In contrast, fitting of the OMA modified by the Hill coefficient to the same data resulted in correct estimates of operational efficacy and affinity. Therefore OMA modified by the Hill coefficient should be preferred over Black et al. equation for ranking of agonism and subsequent analysis, like quantification of signalling bias, when concentration response curves differ in the slope factor.
\end{abstract}

\title{
Keywords
}

Receptor theory; operational model of agonism; operational efficacy; slope factor 


\section{Introduction}

Since its introduction in 1983, the operational model of agonism (OMA) has become a golden standard in an evaluation of agonism and subsequently also of signalling bias ${ }^{1}$. The OMA describes the response of the system as a function of ligand concentration using three parameters: 1) The equilibrium dissociation constant of agonist $\left(K_{A}\right)$ to the receptor initiating functional response; 2) The maximal possible response of the system ( $E_{M A X}$; 3 ) The operational efficacy of agonist $(\tau)$. Original OMA describes the dependence of functional response on the concentration of ligand by rectangular hyperbola (Eq. 2). In practice, positive cooperativity or positive feedback lead to steep and negative cooperativity or negative feedback lead to flat concentration-response curves that the OMA does not fit to. Therefore, an equation intended for the description of non-hyperbolic concentration curves (Eq. 7), particularly for systems where the number of receptors varies, was introduced by Black et al. ${ }^{2}$. Since then, Eq. 7 is commonly used. The presented mathematical analysis of Eq. 7 shows that the slope factor affects the relationship between observed maximal response to agonist and operational efficacy and the relationship between the concentration of agonist for half-maximal response and its equilibrium dissociation constant. The presented case study shows that fitting Eq. 7 results in wrong estimates of model parameters.

The presented case study shows that the use of the Hill coefficient as a slope factor to the original rectangular hyperbola of OMA instead of the slope factor by Black et al. is a viable alternative. The Hill-Langmuir equation was originally formulated to describe the cooperative binding of oxygen molecules to haemoglobin ${ }^{3}$. The Hill coefficient reflects the sense and magnitude of cooperativity among concurrently binding ligands. A value of the Hill coefficient lower than 1 indicates negative cooperativity that is manifested as a flat concentration-response curve. The smaller value of the Hill coefficient the flatter curve is. A value of the Hill coefficient greater than 1 indicates positive cooperativity that is manifested as a steep concentration-response curve. The greater value of the Hill coefficient the steeper curve is. In contrast to Black et al. slope factor, the proposed implementation of the Hill coefficient (Eq. 10) does not affect the centre or asymptotes of the hyperbola describing concentration-response curves. Therefore, the Hill coefficient does not affect relationships among three parameters of the OMA, $\mathrm{K}_{\mathrm{A}}, \mathrm{E}_{\mathrm{MAX}}$ and $\tau$. This makes OMA (Eq. 11) more practical in many ways.

\section{The general concept of the operational model of agonism}

In general, OMA consist of two functions. One function describes the binding of an agonist to a receptor as the dependence of the concentration of agonist-receptor complexes [AR] on the 
concentration of an agonist [A]. The second function describes the dependence of functional response (E) to an agonist on [AR]. OMA expresses the dependence of $\mathrm{E}$ on [A].

\section{Rectangular hyperbolic OMA}

\section{Definition of OMA}

In the simplest case, when both binding and response function are described by rectangular hyperbola (Supplementary Information Figure S1), the resulting function is also rectangular hyperbola. For example, in bi-molecular reaction, the dependence of ligand binding to the receptor $[\mathrm{AR}]$ is described by Eq. 1 where $[\mathrm{A}]$ is the concentration of ligand and $\mathrm{K}_{\mathrm{A}}$ is its equilibrium dissociation constant that represents the concentration of ligand at which half of the total number of receptors, $\mathrm{R}_{\mathrm{T}}$, is bound to the ligand and the other half of the receptors is free.

$$
[A R]=\frac{R_{T}[A]}{[A]+K_{A}} \quad \text { Eq. } 1
$$

If the bound ligand is an agonist, it activates the receptor and produces functional response $\mathrm{E}$. Response as a function of agonist binding (agonist-receptor complexes [AR]) is given by Eq. 2 .

$$
E=\frac{E_{M A X}[A R]}{[A R]+K_{E}} \quad \text { Eq. } 2
$$

Where $E_{M A X}$ is the maximum possible response of the system and $K_{E}$ is the value of [AR] that elicits a half-maximal effect. Various agonists produce a functional response of different strengths. The OMA was postulated to introduce the "transducer ratio" $\tau$ that is given by Eq. 3 .

$$
\tau=\frac{R_{T}}{K_{E}} \quad \text { Eq. } 3
$$

The substitution of Eq. 2 with Eq. 1 and Eq. 3 gives Eq. 4.

$$
E=\frac{[A] \tau E_{M A X}}{[A](\tau+1)+K_{A}} \quad \text { Eq. } 4
$$

Analysis of OMA

Eq.4 is the equation of $\mathrm{OMA}^{1}$. It has three parameters: The equilibrium dissociation constant of agonist $\left(\mathrm{K}_{\mathrm{A}}\right)$ at the response-inducing state of the receptor that is specific to a combination of ligand and receptor. The maximal possible response of the system ( $\mathrm{E}_{\mathrm{MAX}}$ ) is specific to the system. And the "transducer ratio" $(\tau)$ that is specific to a combination of ligand and system. Eq. 4 is a rectangular hyperbola with the horizontal asymptote, the observed maximal response to agonist $\mathrm{A}$ (E'MAX), given by Eq. 5 . 


$$
E_{M A X}^{\prime}=\frac{\tau}{\tau+1} E_{M A X}
$$

A more efficacious agonist (having a high value of parameter $\tau$ ) elicits higher E'MAX than less efficacious agonists (having a low value of parameter $\tau$ ). Thus, $\tau$ is actually operational efficacy. The relationship between parameter $\tau$ and E' ${ }_{M A X}$ is hyperbolic meaning that two highly efficacious agonists (e.g., $\tau$ values 10 and 20) differ in E' ${ }_{M A X}$ values less than two weak agonists (e.g., $\tau$ values 0.1 and 0.2 ).

In Eq. 4, the concentration of agonist A for half-maximal response $\left(\mathrm{EC}_{50}\right)$, is given by Eq. 6 .

$$
E C_{50}=\frac{K_{A}}{\tau+1}
$$

According to Eq. 6 , for $\tau>0$, the $\mathrm{EC}_{50}$ value is always lower than the $\mathrm{K}_{\mathrm{A}}$ value. The $\mathrm{K}_{\mathrm{A}}$ to $\mathrm{EC}_{50}$ ratio is greater for efficacious agonists than for weak agonists. Similarly to E'MAX, the relationship between parameter $\tau$ and $\mathrm{EC}_{50}$ is hyperbolic. In contrast to $\mathrm{E}^{\prime} \mathrm{MAX}$ values, the ratio $\mathrm{K}_{\mathrm{A}}$ to $\mathrm{EC}_{50}$ ratio is more profound for two highly efficacious agonists (e.g., $\tau$ values 10 and 20) than two weak agonists (e.g., $\tau$ values 0.1 and 0.2 ).

\section{Limitations of OMA}

The OMA has several weak points. The major drawback of OMA is the lack of physical basis of the agonist equilibrium dissociation constant $\mathrm{K}_{\mathrm{A}}$. In the Eq. 2 considers agonist binding [AR] refers to agonist binding to the conformation from which the functional response is initiated. The agonist binding to the receptor in an inactive conformation is not observed in the response $\left(\mathrm{K}_{\mathrm{E}} \rightarrow \infty ; \tau=0\right)$ In the radioligand binding experiments agonists bind to all receptor conformations including the inactive ones. For various reasons the receptors in the conformation activating functional response may be scarce or absent from radioligand binding experiments. Then it may be impossible to determine the $\mathrm{K}_{\mathrm{A}}$ value in the radioligand binding experiments. All three parameters of OMA ( $\mathrm{E}_{\mathrm{MAX}}, \mathrm{K}_{\mathrm{A}}$ and $\left.\tau\right)$ are interdependent ${ }^{4}$. To fit Eq. 4 to the experimental data one of the parameters must be fixed. Thus, the maximal response of the system $\mathrm{E}_{\mathrm{MAX}}$ has to be determined before fitting Eq. 4. It can be archived by comparing functional response to a given agonist at system with reduced population of receptors by irreversible alkylation $^{5}$. Another limitation of the OMA is that the shape of the functional response is a rectangular hyperbola. 


\section{Non-hyperbolic OMA}

\section{Definition of non-hyperbolic OMA}

In practice, concentration-response curves steeper or flatter than the ones described by Eq. 4 are observed. In such cases, Eq. 4 does not fit experimental data. As stated by the authors, Eq. 7 was devised for non-hyperbolic dependence of functional response on concentration of agonist $^{2}$.

$E=\frac{[A]^{n} \tau^{n} E_{M A X}}{[A]^{n} \tau^{n}+\left([A]+K_{A}\right)^{n}}$

\section{Analysis of non-hyperbolic OMA}

Introduced power factor $\mathbf{n}$ changes slope and shape of functional response curve (Supplementary information Figure S3). Nevertheless, Eq. 7 as a mathematical function has rectangular asymptotes: The horizontal asymptote $([\mathrm{A}] \rightarrow \pm \infty)$ E' ${ }_{\text {MAX }}$ is given by Eq. 8 and vertical asymptote $(\mathrm{E} \rightarrow \pm \infty)$ is equal to $-\mathrm{K}_{\mathrm{A}} /(\tau+1)$ (Supplementary Information Eq. A2 and Eq. A3). From Eq. 7 , the $\mathrm{EC}_{50}$ value is given by Eq. 9 .

$$
\begin{aligned}
& E^{\prime}{ }_{M A X}=\frac{\tau^{n} E_{M A X}}{1+\tau^{n}} \\
& E C_{50}=\frac{K_{A}}{\left(2+\tau^{n}\right)^{\frac{1}{n}}-1}
\end{aligned}
$$

Evidently, the introduced slope factor $\mathbf{n}$ affects both the observed maximal response E'MAX and half-efficient concentration of agonist $\mathrm{EC}_{50}$ (Figure 1A and C). The influence of the slope factor on E'MAX is bidirectional (Supplementary Information Table S1, Figure S2). For operational efficacies $\tau>1$, an increase in the value of slope factor increases E'MAX. (Figure 1A and 2A blue lines). For operational efficacies $\tau<1$, an increase in slope factor decreases E' ${ }_{\text {MAX }}$ (Figure $1 \mathrm{C}$ and 2A yellow lines). The effect of slope factor on $\mathrm{E}^{\text {' }}{ }_{\mathrm{MAX}}$ is the most eminent for low values of operational efficacy $\tau$, making the estimation of model parameters of weak partial agonists impractical. Imagine full agonist $\tau=10$ and very weak agonist $\tau=0.1$. For $n=1$ : Full agonist E'MAX is $90 \%$ and weak agonist E' ${ }_{\text {MAX }}$ is $10 \%$ of system EMAX. For $n=2$ : Full agonist E' ${ }_{\text {MAX }}$ is $99 \%$ (one-tenth more) and weak agonist E' ${ }_{\text {MAX }}$ is just $1 \%$ (ten times less). 

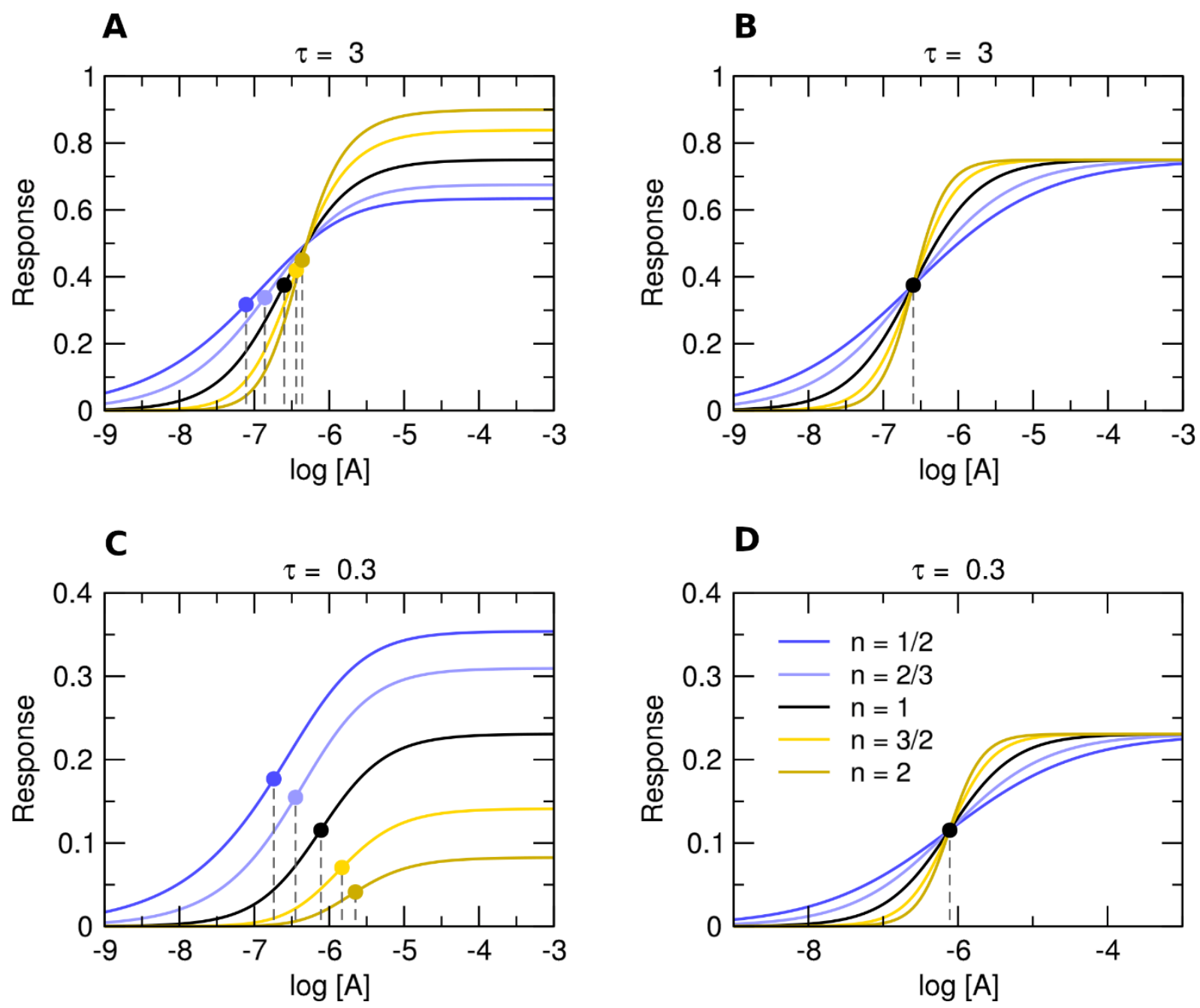

Figure 1 Theoretical concentration curves

Theoretical response curves according to Eq. 7, left and Eq. 11, right. Simulation parameters: $\mathrm{E}_{\mathrm{MAX}}=1 ; \tau=3$ (top) or $\tau=0.3$ (bottom); $\mathrm{K}_{\mathrm{A}}=10^{-6}$. Values of slope factors are listed in the legend.

An increase in the value of the slope factor increases the $\mathrm{EC}_{50}$ value (Figure 2B). Again, the effect of the slope factor on the $\mathrm{EC}_{50}$ value is more eminent at low values of operational efficacy $\tau$ (red lines). Paradoxically, any combination of operational efficacy $\tau$ and slope factor fulfilling the inequality in Figure $2 \mathrm{C}$ (blue area) results in $\mathrm{EC}_{50}$ values greater than $\mathrm{K}_{\mathrm{A}}$ (e.g., Figure $1 \mathrm{C}$, yellow lines). For example, $\mathrm{EC}_{50}>\mathrm{K}_{\mathrm{A}}$ applies if $\tau=0.5$ and $\mathrm{n}>1.6$, or if $\tau=1$ and $\mathrm{n}>1.6$, or if $\tau=1.5$ and $n>2.15$. The upper asymptote of inequality is 2 . Thus, the possibility of $\mathrm{EC}_{50}>\mathrm{K}_{\mathrm{A}}$ applies to $\tau<2$. 
A
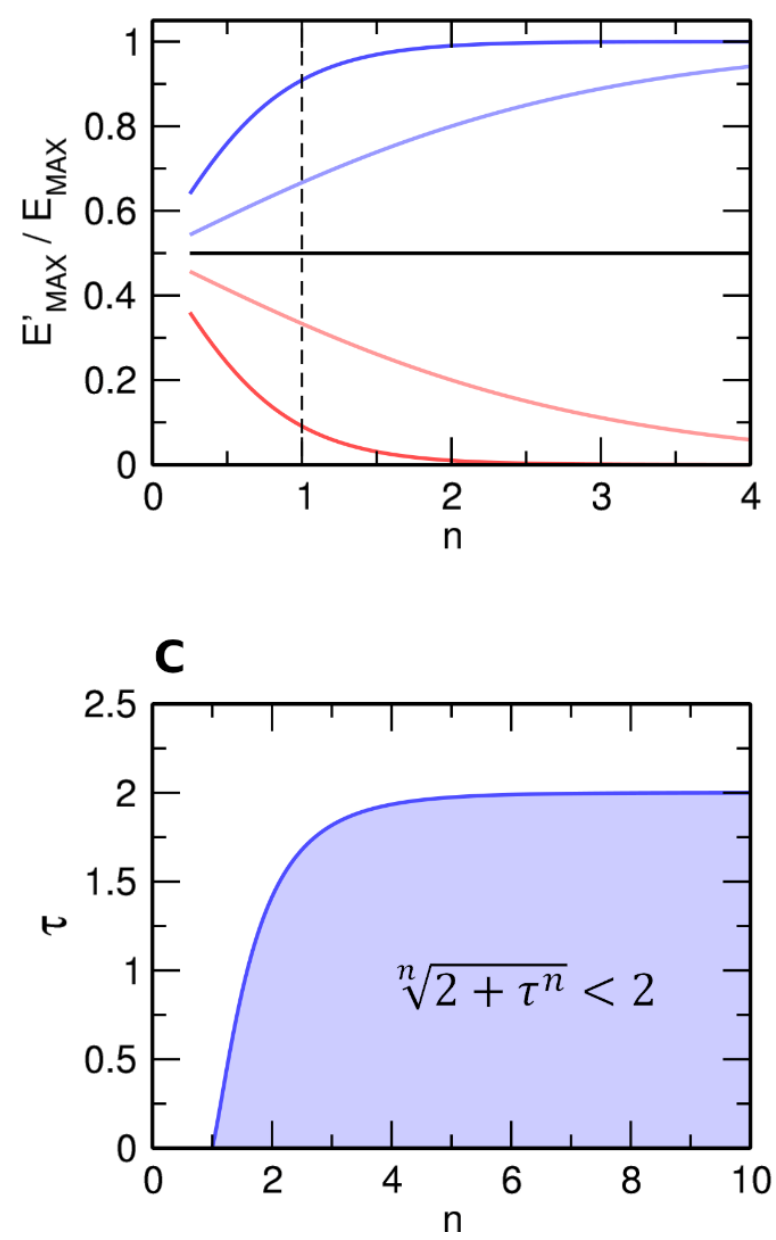

B

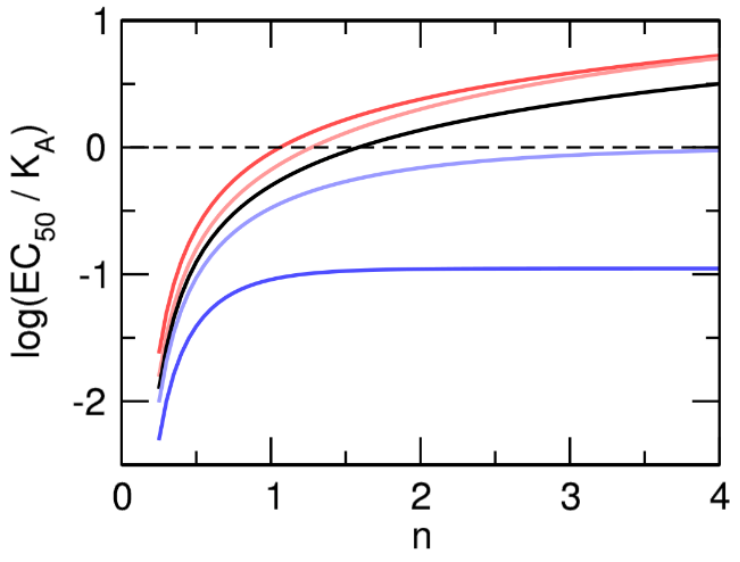

Figure 2 Analysis of Black \& Leff equation (Eq. 7)

A, Dependency of observed E' MAX to system EMAX ratio (ordinate) on slope factor $n$ (abscissa) and operational efficacy $\tau$ (legend). B, Dependency of $\mathrm{EC}_{50}$ to $\mathrm{K}_{\mathrm{A}}$ ratio (ordinate) on slope factor $n$ (abscissa) and operational efficacy $\tau$ (legend). C, Inequality plot of slope factor $\mathbf{n}$ (abscissa) and operational efficacy $\tau$ (ordinate) yielding half-efficient concentration $\mathrm{EC}_{50}$ greater than equilibrium dissociation constant $\mathrm{K}_{\mathrm{A}}$.

The operational efficacy $\tau$ may be also considered as a measure of "receptor reserve". In the system with a relatively small capacity of a functional response output, the full agonist reaches its maximal response before reaching full receptor occupancy. Thus, the agonist $\mathrm{EC}_{50}$ value is lower than its affinity for the receptor. The smaller occupancy fraction is needed for the full response to a given agonist the greater is difference between agonist $\mathrm{EC}_{50}$ and $\mathrm{K}_{\mathrm{A}}$ values. According to OMA (Eq. 2), the relation between $\mathrm{EC}_{50}$ and $\mathrm{K}_{\mathrm{A}}$ is described by Eq. 6 . The greater value of operational efficacy $\tau$, the smaller $\mathrm{EC}_{50}$ value and the greater the difference from $\mathrm{K}_{\mathrm{A}}$. Thus, the value of operational efficacy $\tau$ is a measure of receptor reserve of a given agonist at a given system. In a system with a large capacity of functional output, agonists do not have a receptor reserve and must reach full receptor occupancy to elicit a full signal. In such a system, 
even full agonists have small operational efficacies. Thus, the parameter $\tau$ is specific to a combination of ligand and system.

Nevertheless, for agonists that elicit at least some response in a given system, the parameter $\tau$ must be greater than 0. Then according to Eq.6 of the operational model of agonism, the $\mathrm{EC}_{50}$ value must be smaller than the $\mathrm{K}_{\mathrm{A}}$ value. In principle, the $\mathrm{EC}_{50}$ value greater than the $\mathrm{K}_{\mathrm{A}}$ can be achieved only by some parallel mechanism that increases the apparent $\mathrm{K}^{\prime}{ }_{\mathrm{A}}$, provided that a difference $\mathrm{K}_{\mathrm{A}}$ to $\mathrm{K}_{\mathrm{A}}$ is greater that $\mathrm{EC}_{50}$ to $\mathrm{K}_{\mathrm{A}}$. For example, such mechanism may be negative allosteric modulation of agonist binding or non-competitive inhibiton of functional response.

\section{Limitations of the non-hyperbolic OMA}

Besides all limitations of the hyperbolic OMA, the non-hyperbolic version of OMA has additional drawbacks. The most important is the lack of mechanistic background for factor $\mathbf{n}$. Exponentiation of agonist concentration [A] to power factor $\mathbf{n}$ results in S-shaped curves. As it will be shown later, a combination of common binding mechanisms and functional responses does not result in S-shaped curves. Importantly, as shown above, exponentiation of operational efficacy $\tau$ to power factor $\mathbf{n}$ breaks the logical relationship between observed maximal response E'MAX and operational efficacy $\tau$. That, as it will be shown later, impedes correct estimation of $\tau$ and $K_{\mathrm{A}}$ values.

\section{OMA with Hill coefficient}

\section{Definition of OMA with Hill coefficient}

Hill coefficient may serve as an alternative slope factor in the OMA. Hill equation incorporates the Hill coefficient as a slope factor to rectangular hyperbola ${ }^{3}$. The major advantage of the Hill coefficient as a slope factor is that it allows for a change in the eccentricity (vertices) of the hyperbola-like curves without changing centre $\left(\mathrm{EC}_{50}\right)$ and asymptotes (E'MAX) (Supplementary Information Figure S3). The Hill-Langmuir equation published in 1910 can be formulated as Eq. 10.

$$
E=\frac{[A]^{n H} E^{\prime} M A X}{[A]^{n H}+E C_{50}{ }^{n H}} \quad \text { Eq. } 10
$$

Where $\mathrm{nH}$ is the Hill coefficient. Substitution of E' ${ }_{\mathrm{MAX}}$ by Eq. 5 and $\mathrm{EC}_{50}$ by Eq. 6 gives Eq. 11 .

$$
E=\frac{[A]^{n H} \frac{\tau}{\tau+1} E_{M A X}}{[A]^{n H}+\left(\frac{K A}{\tau+1}\right)^{n H}}
$$


As expected, the Hill coefficient does not influence maximal observed response E' ${ }_{\text {MAX either }}$ half-efficient concentration of agonist $\mathrm{EC}_{50}$ (Figure 1B and D).

\section{Implications of OMA with Hill coefficient}

Analysis of the OMA with slope factor by Black et al. (Eq. 7) have shown that the slope factor $\mathbf{n}$ has a bidirectional effect on the relationship between the parameters E'MAX and $\tau$. and that the slope factor $\mathbf{n}$ affects the relationship between the parameters $\mathrm{EC}_{50}$ and $\mathrm{K}_{\mathrm{A}}$. In contrast, in Eq. 11 neither value of $\mathrm{E}^{\prime}{ }_{\mathrm{MAX}}$ nor the value of $\mathrm{EC}_{50}$ is affected by the Hill coefficient (Figure $1 \mathrm{~B}$ and $\mathrm{D})$. The parameters E'MAX and EC'50 can be readily obtained by fitting Eq. 10 to the single concentration-response data.

\section{Limitations of OMA with Hill coefficient}

The major criticism of the Hill equation is its parsimonious character. It is relatively simple and its parameters are easy to estimate. However, as a model, it is just an approximation. In an experiment, the slope of the concentration-response curve different from unity may be a result of the parallel signalling mechanism providing feedback or allosteric cooperativity. E.g., positive cooperativity results in steep concentration-response curves and negative cooperativity results in flat concentration-response curves.

\section{The case study}

How fitting the Black \& Leff equation to experimental data can affect estimates of the operational efficacy and subsequent analysis is demonstrated on the following example of measurement of the GTP $\gamma \mathrm{S}$ binding as a functional response of $\mathrm{M}_{2}$ receptor to muscarinic agonists carbachol and oxotremorine (Figure 3). At $\mathrm{G}_{\mathrm{i} 1}$ G-protein (Figure 3, left) carbachol stimulated the GTP $\gamma \mathrm{S}$ binding more than oxotremorine. In contrast, at $\mathrm{G}_{\mathrm{OA}}$ G-protein oxotremorine stimulated GTP $\gamma \mathrm{S}$ binding slightly more than carbachol (Figure 3, right). While the functional response to carbachol has normal slope $\left(\mathrm{n}_{\mathrm{H}} \approx 1\right)$, the response to oxotremorine is shallow $\left(\mathrm{n}_{\mathrm{H}}=\right.$ 0.84 and 0.64, respectively) (Table 1). Fitting the Black \& Leff equation (Eq. 10) to the experimental data gives the overlapping curves for carbachol and very similar curves for oxotremorine (Figure 3). Contra intuitively, while at $\mathrm{G}_{\mathrm{OA}}$ the maximal observed response E' ${ }_{\text {MAX }}$ to oxotremorine is higher than E' MAX of carbachol (0.44 vs. 0.42$)$, the estimate of the operational efficacy $\tau$ of oxotremorine is lower than $\tau$ of carbachol (0.55 vs. 0.71). Subsequently, relative intrinsic activity (RAi) ${ }^{6}$ based on $\mathrm{EC}_{50}$ and $\mathrm{E}^{\prime}{ }_{\mathrm{MAX}}$ values for oxotremorine is higher at $\mathrm{G}_{\mathrm{OA}}$ than $\mathrm{G}_{\mathrm{i} 1}$ (21 vs. 18), suggesting slight oxotremorine bias towards $\mathrm{G}_{\mathrm{oA}}$. But bias factor ${ }^{7}$ based on $\tau$ and $\mathrm{K}_{\mathrm{A}}$ values suggest (3.6-fold) bias of oxotremorine towards $\mathrm{G}_{\mathrm{i} 1}$. 

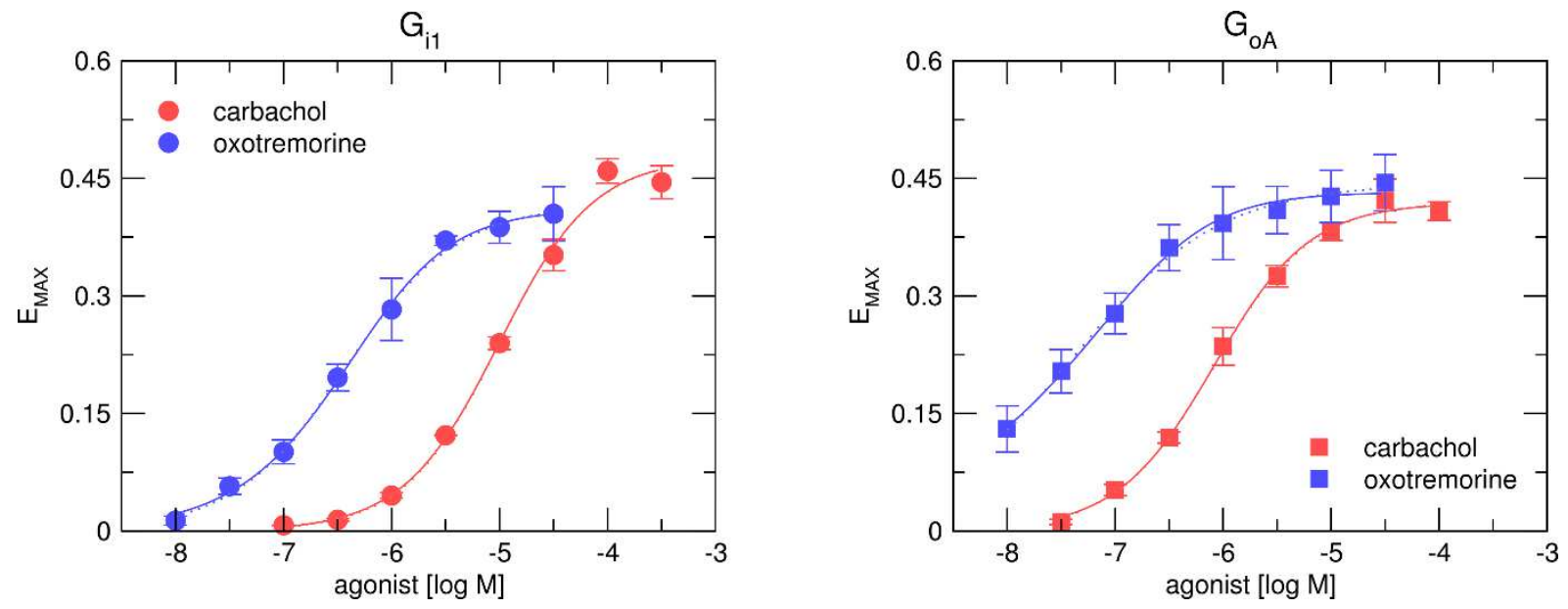

Figure 3 Functional response of muscarinic $M_{2}$ receptors

GTP $\gamma \mathrm{S}$ binding to $\mathrm{G}_{\mathrm{i} 1}$ (left) or $\mathrm{G}_{\mathrm{oA}}$ (right) G-proteins upon stimulation of $\mathrm{M}_{2}$ muscarinic receptors by carbachol (red) or oxotremorine (blue) at the concentration indicated on the $\mathrm{x}$-axis is expressed as the fraction of maximal response of the system ( $\left.\mathrm{E}_{\mathrm{MAX}}\right)$. Curves are from a representative experiment performed in quadruplicates. Solid curves, Hill equation (Eq, 10). Dotted curves, Black \& Leff equation (Eq. 7). Parameter estimates are in Table 1.

Table 1 Results of fitting Black \& Leff and Hill equation to experimental data in Figure 3.

Black\&Leff (Eq. 7) and Hill equation (Eq. 10) were fitted to concentration-response data of GTP $\gamma \mathrm{S}$ binding to G-proteins upon stimulation of $\mathrm{M}_{2}$ receptors by carbachol or oxotremorine in Figure 3. The $\mathrm{E}_{\mathrm{MAX}}$ was fixed to 1.

\begin{tabular}{|c|c|c|c|c|}
\hline & Hill & $\log \mathrm{EC}_{50}$ & $E_{\text {MAX }}^{\prime}$ & $\mathrm{n}_{\mathrm{H}}$ \\
\hline \multirow[t]{2}{*}{$\mathrm{G}_{\mathrm{i} 1}$} & carbachol & $-5.11 \pm 0.06$ & $0.4756 \pm 0.0174$ & $0.99 \pm 0.10$ \\
\hline & oxotremorine & $-6.43 \pm 0.04$ & $0.4167 \pm 0.0090$ & $0.84 \pm 0.05$ \\
\hline \multirow[t]{2}{*}{$\mathrm{G}_{\mathrm{oA}}$} & carbachol & $-6.09 \pm 0.03$ & $0.4204 \pm 0.0068$ & $0.96 \pm 0.06$ \\
\hline & oxotremorine & $-7.39 \pm 0.04$ & $0.4433 \pm 0.0067$ & $0.64 \pm 0.04$ \\
\hline & Black \& Leff & $\log \mathrm{K}_{\mathrm{A}}$ & $\tau$ & $\mathrm{n}$ \\
\hline \multirow[t]{2}{*}{$\mathrm{G}_{\mathrm{i} 1}$} & carbachol & $-4.70 \pm 0.17$ & $0.906 \pm 0.048$ & $0.96 \pm 0.16$ \\
\hline & oxotremorine & $-5.98 \pm 0.10$ & $0.610 \pm 0.027$ & $0.73 \pm 0.07$ \\
\hline \multirow[t]{2}{*}{$\mathrm{G}_{\mathrm{OA}}$} & carbachol & $-5.81 \pm 0.10$ & $0.705 \pm 0.026$ & $0.94 \pm 0.10$ \\
\hline & oxotremorine & $-6.47 \pm 0.13$ & $0.546 \pm 0.036$ & $0.45 \pm 0.05$ \\
\hline
\end{tabular}




\section{OMA of homotropic allosteric modulation}

\section{OMA of homotropic allosteric of binding}

The simplest mode of interaction that leads to variation in the slope of concentration-response curves is allosteric modulation. Let's take for example homotropic allosteric modulation and suppose that ligand A binds to the orthosteric (AR) and the allosteric binding site (RA) with the same affinity. For the sake of simplicity suppose that a ligand A activates the receptor only from the orthosteric binding site. And that A bound to the orthosteric binding site (AR) has the same operational efficacy as A in the ternary complex (ARA) (Figure 4). In such a case, the amount of active complexes AR and ARA is given by Eq. 12 .

$$
[A R]+[A R A]=\frac{R_{T}[A]}{[A]+K_{A} \frac{[A]+K_{A}}{\alpha[A]+K_{A}}}
$$

Where $\alpha$ is the factor of binding cooperativity. Values of $\alpha$ greater than 1 denote positive cooperativity. Values of $\alpha$ smaller than 1 denote negative cooperativity. The substitution of Eq. 12 and Eq. 3 into Eq. 2 gives Eq. 13 (Supplementary Information Eq. A18).

$E=\frac{E_{M A X} \tau[A]\left(K_{A}+\alpha[A]\right)}{K_{A}^{2}+2[A] K_{A}+\alpha[A]^{2}+\tau[A]\left(K_{A}+\alpha[A]\right)}$

Allosteric modulation does not affect observed maximal response E' ${ }_{\text {MAX }}$ (Supplementary Information Eq.A23). For equipotent homotropic allosteric modulation, the value of apparent $\mathrm{K}_{\mathrm{A}}$ $\left(\mathrm{K}^{\prime} \mathrm{A}\right)$ is given by Eq. 14 (Supplementary information Eq. A29) and the value of half-efficient concentration is given by Eq. 15 .

$$
\begin{array}{ll}
K_{A}^{\prime}=\frac{K_{A}}{\sqrt{\alpha}} & \text { Eq. } 14 \\
E C_{50}=\frac{K^{\prime} A}{\tau+1} & \text { Eq. } 15
\end{array}
$$

As can be seen in Figure 4, positive cooperativity results in a steep curve and a decrease in the apparent value of $\mathrm{K}_{\mathrm{A}}$. On the other hand, negative cooperativity results in a flat curve and an increase in the apparent value of $\mathrm{K}_{\mathrm{A}}$. 

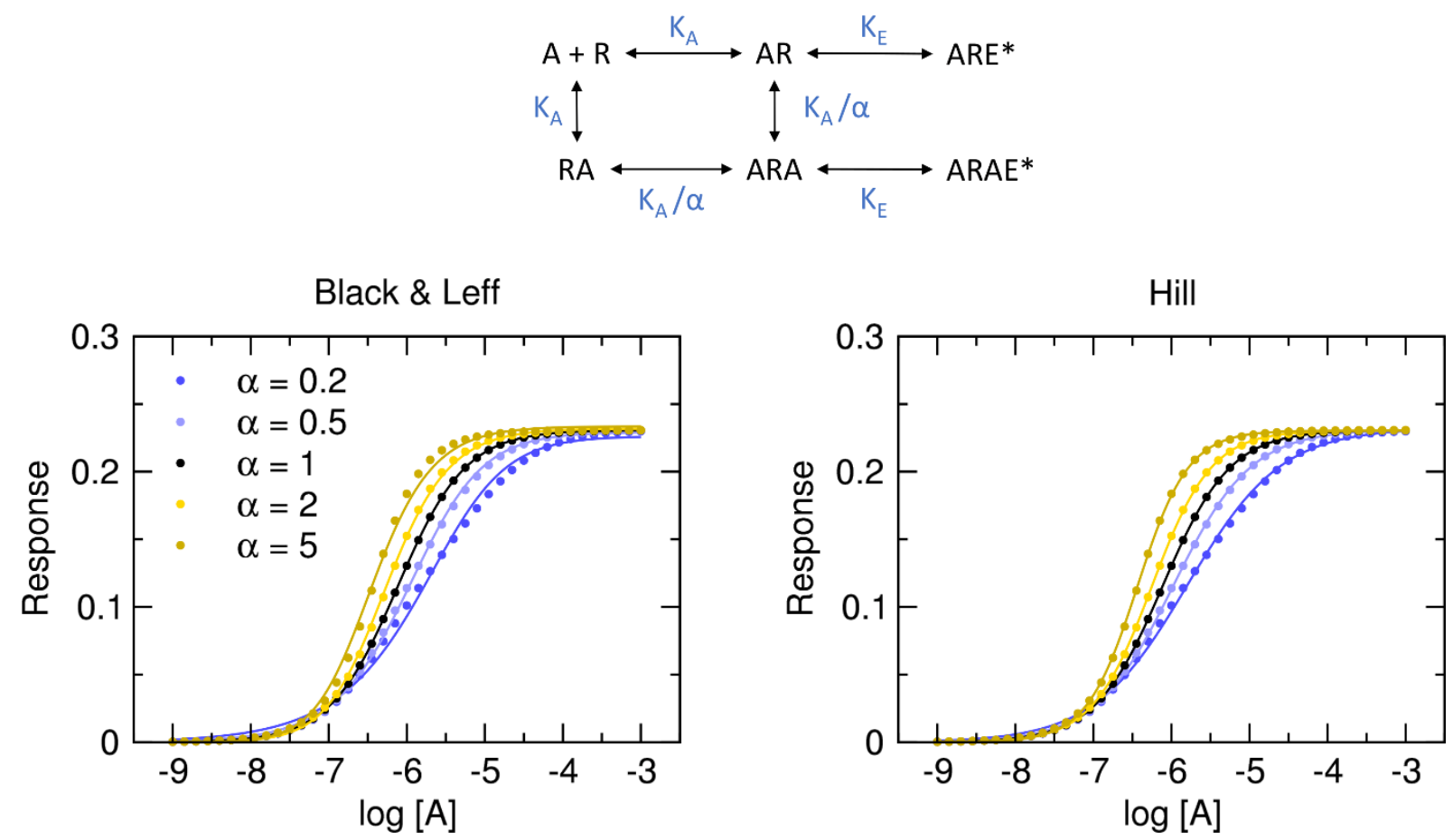

\section{Figure 4 Homotropic allosteric modulation of binding}

Dots, functional response to an agonist $\left(\tau=0.3, \mathrm{E}_{\mathrm{MAX}}=1, \mathrm{R}_{\mathrm{T}}=1\right)$ acting at two sites with the same $\mathrm{K}_{\mathrm{A}}=10^{-6} \mathrm{M}$ exerting binding cooperativity $\alpha$ indicated in the legend, according to Eq. 13. Full lines, left, Black \& Leff equation (Eq. 7), right, Hill equation (Eq. 10) fitted to the data. Parameter estimates are in Table 2.

As can be seen in Figure 3 by the naked eye, the Hill equation fits the data better than the Black \& Leff equation. Importantly, fitting the Hill equation gives correct estimates of apparent maximal response E'MAX, while fitting the Black \& Leff equation results in overestimated E'MAX values in the case of negative cooperativity $(\alpha<1)$ and slightly underestimated E' MAX values in the case of positive cooperativity (Table 2). As result, fitting the Hill equation gives correct estimates of operational efficacies $\tau$. It gives also correct estimates of half-efficient concentrations $\mathrm{EC}_{50}$ and thus correct values of apparent as well as real equilibrium dissociation constant $\mathrm{K}_{\mathrm{A}}$. In contrast, fitting the Black \& Leff equation gives correct parameter estimates only in the case of neutral cooperativity ( $\alpha=1)$. In the case of negative cooperativity $(\alpha<1)$ values of $\tau$ and $\mathrm{pK}_{\mathrm{A}}$ are underestimated. In the case of positive cooperativity $(\alpha>1)$ values of $\tau$ and $\mathrm{pK}_{\mathrm{A}}$ are overestimated. 
Table 2 Results of fitting Black \& Leff and Hill equations to the model of homotropic allosteric modulation of binding

Balck \& Leff (Eq. 7) and Hill (Eq. 10) were fitted to model data (Eq. 13) with EMAX fixed to 1 . Apparent $\mathrm{pK}^{\prime}$ ' A values were calculated from model Eq. 13 according to Eq. 14 or from $\mathrm{EC}_{50}$ values according to Eq. 11.

\begin{tabular}{|c|c|c|c|c|c|c|c|c|c|c|c|}
\hline \multicolumn{4}{|c|}{ Model Eq. 13 and 14} & \multicolumn{3}{c|}{ Black \& Leff } & \multicolumn{5}{c|}{ Hill } \\
\hline $\mathrm{T}$ & $\mathrm{pKA}$ & $\mathrm{a}$ & pK'A & $\mathrm{n}$ & $\mathrm{T}$ & $\mathrm{pKA}$ & $\mathrm{nH}$ & $\mathrm{EC} 50$ & $\mathrm{E}^{\prime} \mathrm{MAX}$ & $\mathrm{T}$ & $\mathrm{pK} \mathrm{A}$ \\
\hline 0.3 & 6.00 & 0.2 & 5.65 & 0.59 & 0.123 & 5.30 & 0.72 & 5.80 & 0.23 & 0.3 & 5.65 \\
\hline 0.3 & 6.00 & 0.5 & 5.85 & 0.78 & 0.210 & 5.69 & 0.87 & 5.98 & 0.23 & 0.3 & 5.85 \\
\hline 0.3 & 6.00 & 1.0 & 6.00 & 1.00 & 0.300 & 6.00 & 1.00 & 6.11 & 0.23 & 0.3 & 6.00 \\
\hline 0.3 & 6.00 & 2.0 & 6.15 & 1.35 & 0.411 & 6.32 & 1.14 & 6.25 & 0.23 & 0.3 & 6.15 \\
\hline 0.3 & 6.00 & 5.0 & 6.35 & 1.54 & 0.461 & 6.58 & 1.33 & 6.44 & 0.23 & 0.3 & 6.35 \\
\hline
\end{tabular}

Fitting Eq. 13 with fixed system $\mathrm{E}_{\mathrm{MAX}}$ to the model of functional response homotropic allosteric modulation of binding yields correct parameter estimates that are associated with the low level of uncertainty (Supplementary Information Figure S6).

OMA of homotropic allosteric modulation of efficacy

Homotropic allosteric modulation of agonist binding may result in the change in operational efficacy of agonist $\tau$ (Figure 5). If the operational efficacy of AR is $\tau$ and the operational efficacy of ARA is $\beta \tau\left(K_{E} / \beta\right)$ then the apparent activation constant $K^{\prime}{ }_{E}$ changes with the concentration of an agonist according to Eq. 16.

$K_{E}^{\prime}=K_{E} \frac{[A]+K_{A}}{\beta[A]+K_{A}}$

Eq. 16

Where $\beta$ is the cooperativity factor of efficacy. Values of $\beta$ greater than 1 denote positive cooperativity, a decrease in apparent K'E. Values of $\beta$ smaller than 1 denote negative cooperativity, an increase in apparent $\mathrm{K}_{\mathrm{E}}{ }^{\mathrm{N}}$.

For the sake of simplicity suppose neutral cooperativity $(\alpha=1)$ in the binding of A to the orthosteric and allosteric sites. Then agonist binding described by Eq. 12 simplifies to Eq. Substitution of $\mathrm{K}_{\mathrm{E}}$ by Eq. 16 and ([AR] + [ARA]) by Eq. 1 in Eq. 2 gives Eq. 17 (Supplementary Information Eq. A34).

$E=\frac{[A] \tau E_{M A X}\left(\beta[A]+K_{A}\right)}{K_{A}^{2}+2[A] K_{A}+[A]^{2}+\tau[A]\left(\beta[A]+K_{A}\right)}$

The apparent maximal response E'MAX is given by Eq. 18 (Supplementary Information Eq. A39). 


$$
E_{M A X}^{\prime}=E_{M A X} \frac{\beta \tau}{1+\beta \tau}
$$

Thus, apparent operational efficacy $\tau$ ' is given by Eq. 19 .

$\tau^{\prime}=\beta \tau$

Eq. 19

The $\mathrm{EC}_{50}$ value in homotropic allosteric modulation of efficacy is given by Eq. 20 (Supplementary information Eq. A42).

$E C_{50}= \pm \frac{\sqrt{K_{A}^{2}\left(4 \beta^{2} \tau+\beta^{2}\left(\tau^{2}-4 \tau+8\right)+4 \beta(\tau-2)+4\right)} \pm K_{A}(\beta(\tau-2)+2)}{2 \beta(1+\beta \tau)}$

Both positive $(\beta>1)$ and negative $(\beta<1)$ cooperativity in operational efficacy lead to steep concentration-response curves (Figure 5, Table 3). Obviously, the Hill equation fits the data better than the Black \& Leff equation. Strong negative cooperativity ( $\beta=0.2$ ) results in bellshaped concentration-response curve (Figure 5, dark blue). Except for the bell-shaped curve ( $\beta=0.2)$, fitting of the Hill equation gives correct estimates of apparent maximal response E'MAX and thus correct estimates of apparent operational efficacy $\tau$ '. In contrast, fitting the Black \& Leff equation results in overestimated $\tau$ values. In the case of positive cooperativity $(\beta>1), \tau$ values are close to apparent operational efficacy $\tau^{\prime}$. Except for $\beta=2, \mathrm{pK}_{\mathrm{A}}$ values are overestimated. 


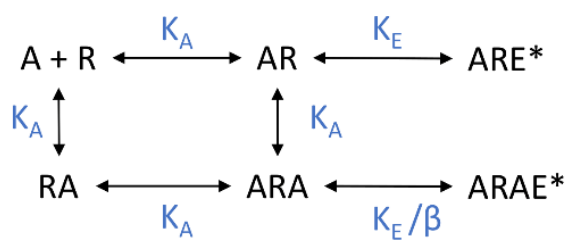

Black \& Leff

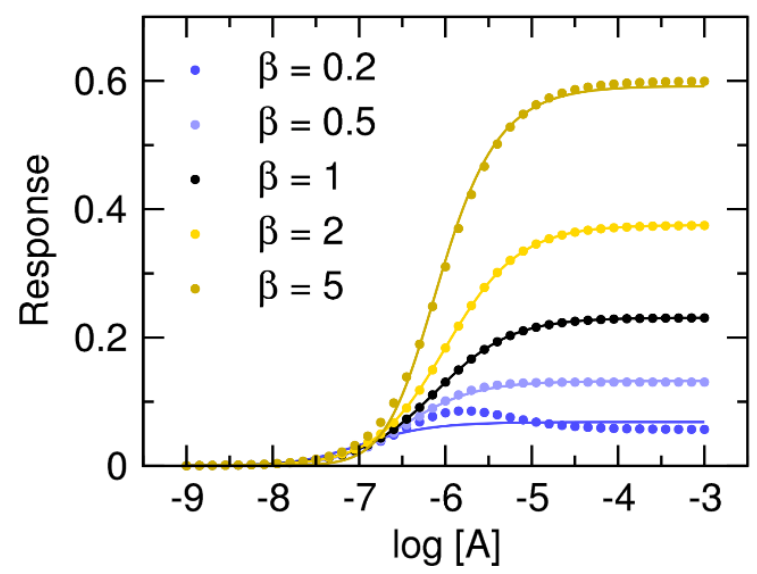

Hill

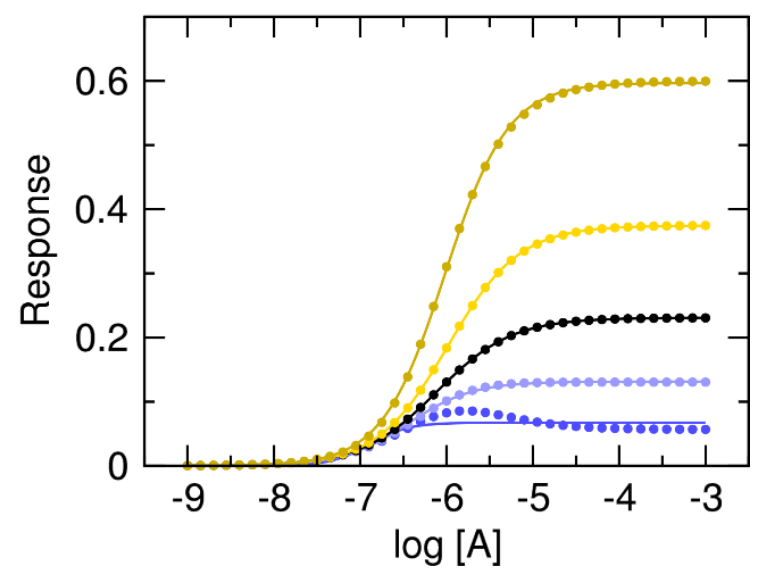

Figure 5 Homotropic allosteric modulation of efficacy

Dots, functional response to an agonist ( $\tau=0.3, \mathrm{E}_{\mathrm{MAX}}=1, \mathrm{R}_{\mathrm{T}}=1$ ) acting at two sites with the same $K_{A}=10^{-6} \mathrm{M}$ exerting cooperativity in efficacy $\beta$ indicated in the legend, according to Eq. 17. Full lines, left, Black \& Leff equation (Eq. 7), right, Hill equation (Eq. 10) fitted to the data. Parameter estimates are in Table 3.

Table 3 Results of fitting Black \& Leff and Hill equations to the model of homotropic allosteric modulation of efficacy

Black \& Leff (Eq. 7) and Hill (Eq. 10) equations were fitted to model data (Eq. 17) with EMAX fixed to 1 .

\begin{tabular}{|c|c|c|c|c|c|c|c|c|c|c|}
\hline \multicolumn{4}{|c|}{ Model Eq. 17 and 19 } & \multicolumn{3}{c|}{ Black \& Leff } & \multicolumn{3}{c|}{ Hill } \\
\hline $\mathrm{T}$ & $\mathrm{pK} \mathrm{A}_{\mathrm{A}}$ & $\beta$ & $\mathrm{T}$ & $\mathrm{n}$ & $\mathrm{T}$ & $\mathrm{pK}$ & $\mathrm{n} H$ & $\mathrm{EC}_{50}$ & $\mathrm{E}^{\prime}$ MAx & $\mathrm{T}^{\prime}$ \\
\hline 0.3 & 6.00 & 0.2 & 0.06 & 1.48 & 0.171 & 7.15 & 1.93 & 6.89 & 0.067 & 0.072 \\
\hline 0.3 & 6.00 & 0.5 & 0.15 & 1.35 & 0.248 & 6.57 & 1.16 & 6.45 & 0.131 & 0.151 \\
\hline 0.3 & 6.00 & 1.0 & 0.30 & 1.00 & 0.300 & 6.00 & 1.00 & 6.11 & 0.230 & 0.300 \\
\hline 0.3 & 6.00 & 2.0 & 0.60 & 1.12 & 0.636 & 5.86 & 1.06 & 5.98 & 0.374 & 0.598 \\
\hline 0.3 & 6.00 & 5.0 & 1.50 & 2.05 & 1.199 & 6.14 & 1.19 & 6.02 & 0.597 & 1.481 \\
\hline
\end{tabular}

Fitting Eq. 17 with fixed system $\mathrm{E}_{\mathrm{MAX}}$ to the model of functional response homotropic allosteric modulation of efficacy yields correct parameter estimates that are associated with the low level of uncertainty, except for neutral cooperativity $(\beta=1)$ (Supplementary Information Figure S7). This should not represent a problem as for neutral cooperativity $(\beta=1)$ Hill coefficient equals one and thus OMA in the form of Eq. 4 can be used. 


\section{OMA of substrate inhibition}

Another common mechanism affecting the functional response to an agonist is substrate inhibition. In substrate inhibition, substrate concentration-dependently inhibits the reaction. In the case of functional response to receptor activation, agonist-receptor complexes [AR] are the substrate (Figure 6). Functional response is given by Eq. 21.

$E=E_{M A X} \frac{[A R]}{K_{E}+[A R]\left(1+\frac{[A R]}{K_{I}}\right)}$

Where $\mathrm{K}_{\mathrm{I}}$ is the inhibition constant, the concentration of [AR] that causes $50 \%$ inhibition of the functional response. After substitution of Eq. 1 for [RA], Eq. 21 becomes Eq. 22 (Supplementary information Eq. A50).

$E=E_{M A X} \frac{[A] \tau\left([A]+K_{A}\right)}{\sigma \tau[A]^{2}+\left([A]+K_{A}\right)^{2}+[A] \tau\left(A+K_{A}\right)}$

Where $\sigma=\mathrm{R}_{\mathrm{T}} / \mathrm{K}_{\mathrm{I}}$. The apparent maximal response E' ${ }_{\mathrm{MAX}}$ is given by Eq. 23 (Supplementary Information Eq. A46).

$E_{M A X}^{\prime}=E_{M A X} \frac{\tau}{1+\tau(1+\sigma)}$

Eq. 23

Thus, apparent operational efficacy $\tau$ ' is given by Eq. 24 .

$\tau^{\prime}=\frac{\tau}{\sigma \tau+1}$

The $\mathrm{EC}_{50}$ value for the model of substrate inhibition is given by Eq. 25 (Supplementary information Eq. A53).

$E C_{50}= \pm \frac{\sqrt{\sigma^{2} \tau^{2}+4 \sigma \tau^{2}+8 \sigma \tau+\tau^{2}+16 \tau+8}-2 K_{A} \sigma \tau-K_{A} \tau}{2(\sigma \tau+2 \tau+1)}$

The shape for functional response is similar to homotropic allosteric modulation of efficacy (Figure 6). Again, the Hill equation fits the data better than the Black \& Leff equation. For strong inhibition $(\sigma=10)$ curves of the functional response have a bell shape. Except for the bellshaped curve, fitting of the Hill equation gives correct estimates of apparent maximal response E'MAX and thus correct estimates of apparent operational efficacy $\tau$ '. In contrast, fitting the Black \& Leff equation results in underestimated $\tau$ values and overestimated $\mathrm{pK}_{\mathrm{A}}$ values. Stronger inhibition (greater value of $\sigma$ ) results in the larger overestimation of $\mathrm{pK}_{\mathrm{A}}$. 


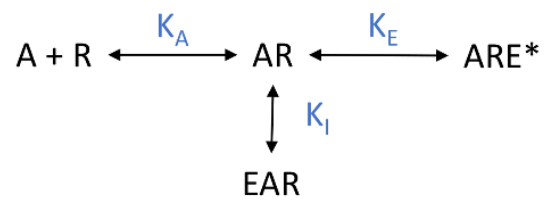

Black \& Leff
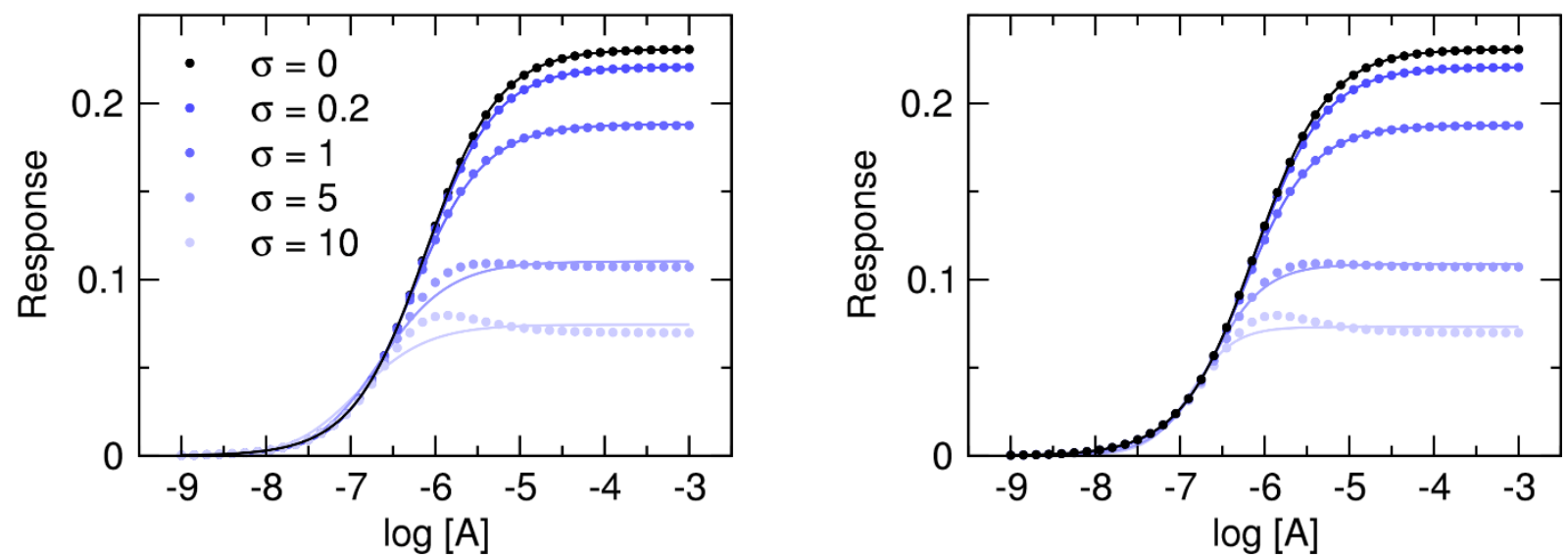

Figure 6 Substrate inhibition of functional response

Dots, functional response to an agonist $\left(\tau=0.3, \mathrm{E}_{\mathrm{MAX}}=1, \mathrm{R}_{\mathrm{T}}=1, \mathrm{~K}_{\mathrm{A}}=10^{-6} \mathrm{M}\right)$ in the model of substrate inhibition according to Eq. 22. Values of $\sigma$ are indicated in the legend. Full lines, left, Black \& Leff equation (Eq. 7), right, Hill equation (Eq. 10) fitted to the data. Parameter estimates are in Table 4.

Table 4 Results of fitting Black \& Leff and Hill equations to the model of substrate inhibition

Black \& Leff (Eq. 7) and Hill (Eq. 10) were fitted to model data Eq. 22 with EMAX fixed to 1 .

\begin{tabular}{|c|c|c|c|c|c|c|c|c|c|c|}
\hline \multicolumn{4}{|c|}{ Model Eq. 22 and 24 } & \multicolumn{3}{c|}{ Black \& Leff } & \multicolumn{4}{c|}{ Hill } \\
\hline $\mathrm{T}$ & $\mathrm{pK}_{\mathrm{A}}$ & $\sigma$ & $\mathrm{T}^{\prime}$ & $\mathrm{n}$ & $\mathrm{T}$ & $\mathrm{pK}_{\mathrm{A}}$ & $\mathrm{n}_{\mathrm{H}}$ & $\mathrm{EC}_{50}$ & $\mathrm{E}^{\prime}$ MAX & $\mathrm{T}^{\prime}$ \\
\hline 0.3 & 6.00 & 0 & 0.300 & 1.00 & 0.300 & 6.00 & 1.01 & 6.00 & 0.226 & 0.300 \\
\hline 0.3 & 6.00 & 0.5 & 0.283 & 1.02 & 0.292 & 6.05 & 1.01 & 6.15 & 0.221 & 0.283 \\
\hline 0.3 & 6.00 & 1 & 0.231 & 1.14 & 0.276 & 6.25 & 1.06 & 6.26 & 0.188 & 0.231 \\
\hline 0.3 & 6.00 & 5 & 0.120 & 1.37 & 0.218 & 6.76 & 1.37 & 6.63 & 0.109 & 0.121 \\
\hline 0.3 & 6.00 & 10 & 0.075 & 1.37 & 0.159 & 7.04 & 1.72 & 6.87 & 0.073 & 0.079 \\
\hline
\end{tabular}

Fitting Eq. 22 with fixed system EMAX to the model of functional response of substrate inhibition yields correct parameter estimates that are associated with the low level of uncertainty (Supplementary Information Figure S8).

\section{OMA of non-competitive inhibition}

As shown in Figure 2, OMA with slope factor $\mathbf{n}$ allows for $\mathrm{EC}_{50}$ values higher than $\mathrm{K}_{\mathrm{A}}$. The simplest mode of interaction that increases observed $\mathrm{EC}_{50}$ above $\mathrm{K}_{\mathrm{A}}$ is non-competitive autoin- 
hibition. Under non-competitive auto-inhibition, AR non-competitively blocks functional response (Figure 7). It results in a concentration-dependent increase in $\mathrm{EC}_{50}$ and a decrease in E'MAX. Functional response is given by Eq. 26.

$E=\frac{E_{M A X}[A R]}{\left(K_{E}+[A R]\right) \frac{[A R]}{K_{I}+[A R]}}$

For $\mathrm{K}_{\mathrm{I}}>0$, after substitution of Eq. 1 for [RA], Eq. 26 becomes Eq. 27 (Supplementary information Eq. A57).

$E=E_{M A X} \frac{\tau[A]}{K_{A}+[A](1+\tau)} \frac{\sigma[A]}{K_{A}+[A](1+\sigma)}$

Where $\sigma=\mathrm{R}_{\mathrm{T}} / \mathrm{K}_{\mathrm{I}}$. The apparent maximal response E' ${ }_{\text {MAX }}$ is given by Eq. 28 (Supplementary Information Eq. A57).

$E_{M A X}^{\prime}=E_{M A X} \frac{\tau}{1+\tau} \frac{\sigma}{1+\sigma}$

Thus, apparent operational efficacy $\tau$ ' is given by Eq. 29 .

$\tau^{\prime}=\frac{\sigma \tau}{\sigma+\tau+1}$

The $\mathrm{EC}_{50}$ value for the model of non-competitive auto-inhibition is given by Eq. 30 (Supplementary information Eq. A61).

$E C_{50}=K_{A} \frac{\sqrt{\sigma^{2}+6 \sigma \tau+8 \sigma+\tau^{2}+8 \tau+8}+\sigma+\tau+2}{2(1+\sigma)(1+\tau)}$

Non-competitive auto-inhibition decreases apparent maximal response E' ${ }_{\mathrm{MAX}}$, increases observed half-efficient concentration $\mathrm{EC}_{50}$ and results in steep curves (Figure 7). Resulting concentration-response curve is asymmetric with a typical slope factor about 1.2 regardless of values of $\mathrm{K}_{\mathrm{I}}$ and $\mathrm{K}_{\mathrm{E}}$. Both models fit well. Fitting Hill equation gives correct estimates of apparent maximal response E'MAX and thus correct estimates of apparent operational efficacy $\tau$ ' (Table 5). In contrast, for $K_{I} \geq 1$, fitting the Black \& Leff equation results in underestimated values of $\tau$ and $\mathrm{pK}_{\mathrm{A}}$. 


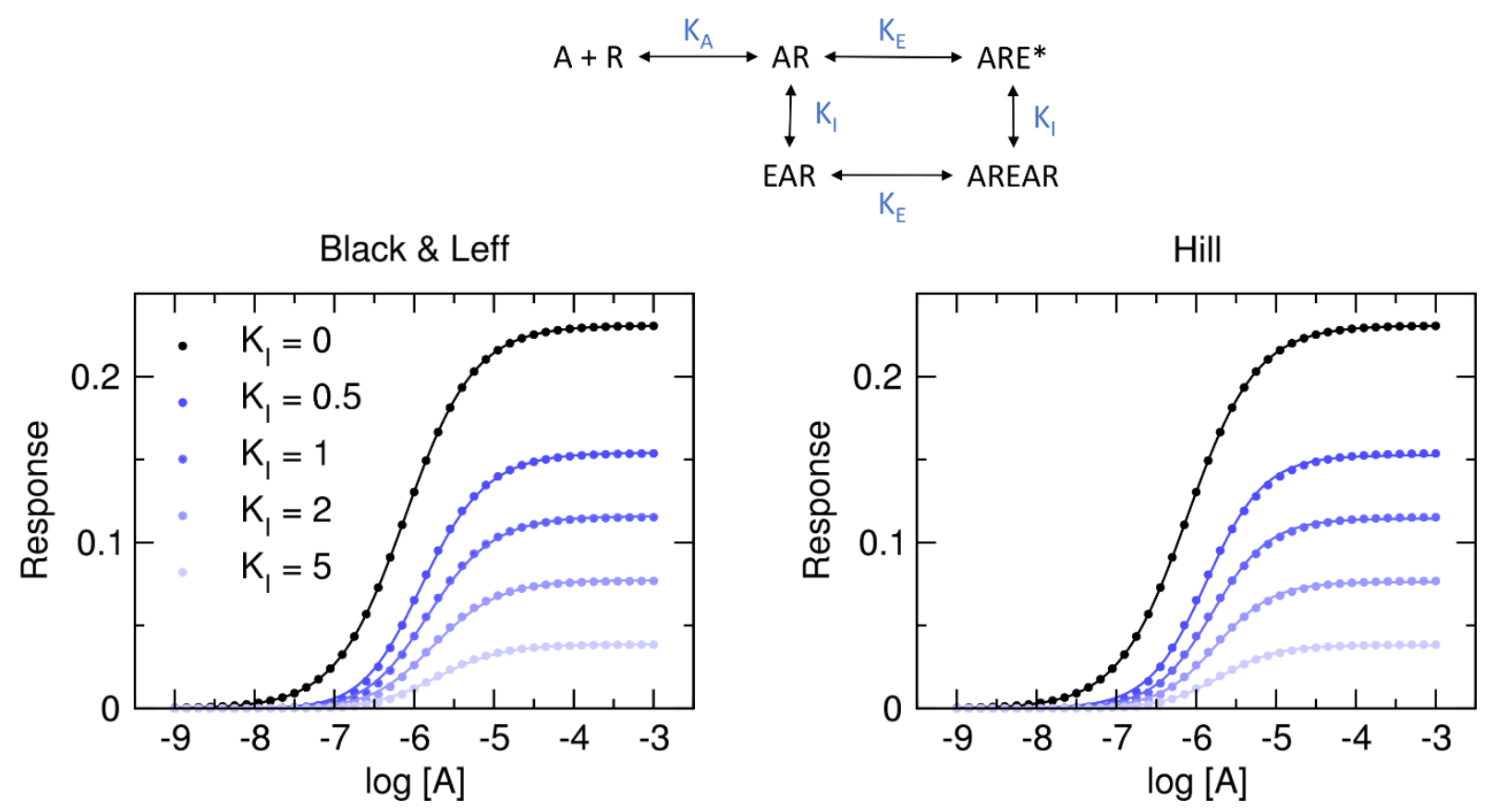

Figure 7 Non-competitive auto-inhibition of functional response

Dots, functional response to an agonist $\left(\tau=0.3, \mathrm{E}_{\mathrm{MAX}}=1, \mathrm{R}_{\mathrm{T}}=1, \mathrm{~K}_{\mathrm{A}}=10^{-6} \mathrm{M}\right)$ in the model of non-competitive autoinhibition according to Eq. 27. Values of inhibition constants $\mathrm{K}_{\mathrm{I}}$ are indicated in the legend. Full lines, left, Black \& Leff equation (Eq. 7), right, Hill equation (Eq. 10) fitted to the data. Parameter estimates are in Table 5.

Table 5 Results of fitting Black \& Leff equations to the model of non-competitive auto-inhibition

Black \& Leff (Eq. 7) and Hill (Eq. 10) equations were fitted to model data Eq. (27) with $\mathrm{E}_{\mathrm{MAX}}$ fixed to 1 .

\begin{tabular}{|c|c|c|c|c|c|c|c|c|c|c|}
\hline \multicolumn{4}{|c|}{ Model Eq. 27 and 29 } & \multicolumn{3}{c|}{ Black \& Leff } & \multicolumn{4}{c|}{ Hill } \\
\hline $\mathrm{T}$ & $\mathrm{pK}_{\mathrm{A}}$ & $\mathrm{K}_{\mathrm{I}}$ & $\mathrm{T}^{\prime}$ & $\mathrm{n}$ & $\mathrm{T}$ & $\mathrm{pK}_{\mathrm{A}}$ & $\mathrm{n}_{\mathrm{H}}$ & $\mathrm{EC}_{50}$ & $\mathrm{E}_{\text {'MAX }}$ & $\mathrm{T}^{\prime}$ \\
\hline 0.3 & 6.00 & 0 & 0.300 & 1.00 & 0.300 & 6.00 & 1.00 & 6.11 & 0.231 & 0.300 \\
\hline 0.3 & 6.00 & 0.5 & 0.182 & 1.44 & 0.307 & 6.02 & 1.20 & 5.87 & 0.153 & 0.181 \\
\hline 0.3 & 6.00 & 1 & 0.130 & 1.38 & 0.230 & 5.94 & 1.21 & 5.81 & 0.114 & 0.129 \\
\hline 0.3 & 6.00 & 2 & 0.083 & 1.32 & 0.154 & 5.87 & 1.22 & 5.75 & 0.076 & 0.082 \\
\hline 0.3 & 6.00 & 5 & 0.040 & 1.25 & 0.077 & 5.8 & 1.22 & 5.70 & 0.038 & 0.040 \\
\hline
\end{tabular}

Fitting Eq. 27 with fixed system $E_{\text {MAX }}$ to the model of functional response of substrate inhibition yields correct parameter estimates that are associated with the low level of uncertainty only when correct initial estimates of $\tau$ and $\sigma$ are given (Supplementary Information Figure S9 and S10). In the case of $\mathrm{K}_{\mathrm{I}}=5$ (Supplementary Information Figure S9), estimates of operational efficacy $\tau$ and inhibition factor $\sigma$ are swapped pointing to the symmetry of Eq. 27. This symmetry makes calculation of $\tau$ and $\sigma$ impossible as any $\tau$ and $\sigma$ combination resulting in an appropriate apparent efficacy $\tau^{\prime}$ (Eq. 28) fits well a given functional-response data (Supplementary Information Figure S10). 


\section{Discussion}

The operational model of agonism (OMA $)^{1}$ is widely used in the evaluation of agonism. The OMA characterizes a functional response to an agonist by the equilibrium dissociation constant of agonist $\left(\mathrm{K}_{\mathrm{A}}\right)$, the maximal possible response of the system ( $\left.\mathrm{E}_{\mathrm{MAX}}\right)$ and the operational efficacy of agonist $(\tau)$ (Eq. 4). To fit non-hyperbolic functional responses slope factor $n$ was introduced to the OMA (Eq. 7) ${ }^{2}$. Analysis of the Black \& Leff equation (Eq. 7) has shown that the slope factor $\mathbf{n}$ has a bidirectional effect on the relationship between the parameters E'MAX and $\tau$ (Figure 1A versus 1C) and also affects the relationship between the parameters $\mathrm{EC}_{50}$ and $\mathrm{K}_{\mathrm{A}}$. In practice, as exemplified in Figure 3, fitting the Black \& Leff equation may result in operational efficacy $\tau$ lower than $\tau$ of control agonist although agonist exerts higher E'MAX than control agonist. This may lead to wrong agonist ranking and compromise subsequent analysis and conclusions. Fitting Black \& Leff equation to the models of homotropic allosteric modulation of affinity (Figure 4), homotropic allosteric modulation of efficacy (Figure 5), substrate inhibition (Figure 6) and non-competitive auto-inhibition (Figure 7) also resulted in wrong estimates of $\tau$ and $\mathrm{K}_{\mathrm{A}}$ values (Tables 2 to 5).

Biased agonists stabilize specific conformations of the receptor leading to non-uniform modulation of individual signalling pathways ${ }^{8}$. To measure an agonist bias, the parameters $\tau$ and $\mathrm{K}_{\mathrm{A}}$ must be determined and $\log \left(\tau / \mathrm{K}_{\mathrm{A}}\right)$ values of tested and reference agonist compared at two signalling pathways ${ }^{9}$. It is evident from the analysis of the Black \& Leff equation, that as far as the $\mathrm{EC}_{50}$ value is dependent on parameters $\mathbf{n}$ and $\tau$ (Figure $1 \mathrm{~A}$ vs. $\mathrm{C}$ ), $\log \left(\tau / \mathrm{K}_{\mathrm{A}}\right)$ values cannot be compared to judge possible signalling bias unless the parameter $\mathbf{n}$ is equal to 1 for both tested and reference agonist.

Despite the dire effects of slope factor $\mathbf{n}$, the Black \& Leff equation is widely accepted ${ }^{7,10-16}$. It even entered textbooks ${ }^{17}$. Very little concern on factor $\mathbf{n}$ has risen. For example, Kenakin et al. ${ }^{9}$ analysed in detail the effects of slope factor $\mathbf{n}$ on $\mathrm{EC}_{50}$ and $\tau$ to $\mathrm{K}_{\mathrm{A}}$ ratio but did not deal with the bi-directional effect of $\mathbf{n}$ on $\tau$ neither proposed an alternative approach to avoid potential pitfalls. To force a proper shape on functional-response curves, Gregory et al. ${ }^{18}$ introduced the second slope factor into their OMA and operational model of allosterically modulated agonism (OMAMA) analysis making equations even more complex. So far, the greatest criticism of OMA was voiced by Roche et al. ${ }^{19}$, noting that to accommodate the shape of theoretical curves Black \& Leff equation tends to overestimate equilibrium dissociation constant $\mathrm{K}_{\mathrm{A}}$ and 
operational efficacy $\tau$ and thus be misleading. They advocated for different expressions of operational models including OMA modified by Hill coefficient in the case of symmetric concentration-response curves.

The fitting of the Hill equation (Eq. 10) to the functional response is straightforward and easier than fitting the Black \& Leff equation. As shown in Figures 3 to 6, the Hill equation fits well to various functional-response curves, often better than the Black \& Leff equation. In contrast to the Black \& Leff equation, the Hill equation gives correct estimates of maximal response to agonist $\mathrm{E}^{\prime}{ }_{\mathrm{MAX}}$ and its half-efficient concentration $\mathrm{EC}_{50}$ as documented by Tables 1 to 5 . In the case of the Hill equation, neither value of $\mathrm{E}^{\prime}{ }_{\mathrm{MAX}}$ nor the value of $\mathrm{EC}_{50}$ is affected by the Hill coefficient (Figure 1B and D). Therefore, biased signalling may be inferred from the comparison of the ratio of intrinsic activity $\left(\mathrm{E}^{\prime} \mathrm{MAX}_{\mathrm{X}} / \mathrm{EC}_{50}\right)$ of tested agonist to the intrinsic activity of reference agonist at two signalling pathways as in the case of Hill equation the E' ${ }_{\mathrm{MAX}} / \mathrm{EC}_{50}$ ratio is equivalent to $\tau / \mathrm{K}_{\mathrm{A}}$ ratio $^{6,20}$. As shown for experimental data in Figure 3 and Table 1, relative intrinsic activities reveal signalling bias even when calculation bias factor based on $\tau$ and $\mathrm{K}_{\mathrm{A}}$ values estimated by Black \& Leff equation gives an incorrect conclusion.

Further, if needed, apparent operational efficacy $\tau^{\prime}$ can be calculated from known E'MAX values and known maximal response of the system $\mathrm{E}_{\mathrm{MAX}}$. From the relationship between $\tau$ ' and $\mathrm{EC}_{50}{ }^{4,21}$ the mechanism of functional response can be inferred by comparison to explicit models. Four examples of explicit models are given above. Subsequently, equilibrium dissociation constant $\mathrm{K}_{\mathrm{A}}$ and operational efficacy $\tau$ alongside the rest of model-specific parameters can be obtained by fitting the explicit model to experimental data.

\section{Conclusions}

Analysis of the Black \& Leff equation has shown that i) The slope factor $\mathbf{n}$ has a bidirectional effect on the relationship between the parameters E'MAX and $\tau$. ii) The slope factor $\mathbf{n}$ affects the relationship between the parameters $\mathrm{EC}_{50}$ and $\mathrm{K}_{\mathrm{A}}$. Fitting the Black \& Leff equation gives wrong estimates of $\tau$ and $\mathrm{K}_{\mathrm{A}}$ values when slope factor $\mathbf{n}$ differs from unity, limiting the use of the Black \& Leff equation in the evaluation of signalling bias. Analysis of the Hill equation has shown that the Hill coefficient does not affect the relationship between the parameters E'MAX and $\tau$ neither between the parameters $\mathrm{EC}_{50}$ and $\mathrm{K}_{\mathrm{A}}$. Fitting Hill equation to the concentrationresponse data gives good estimates of $\mathrm{EC}_{50}$ and $\mathrm{E}_{\text {MAX }}$ values, which are suitable for further analysis of OMA, OMAMA and signalling bias, e.g. using relative intrinsic activities. 


\section{Methods}

Preparation of cells and membranes

Spodoptera frugiperda cells (Sf9) (Gibco) were maintained as a suspension culture in serumfree insect cell growth medium SF900 III (Gibco) in a plastic Erlenmeyer flask in a shaking incubator at $27^{\circ} \mathrm{C}$ and $135 \mathrm{rpm}$ in a non-humidified environment. The cultures were maintained at a density of 1-4 $10^{6}$ cells $/ \mathrm{ml}$. The density of the cells was determined with a haemocytometer, and viability was assessed by the exclusion of $0.2 \%$ trypan blue (Sigma-Aldrich). Human $\mathrm{M}_{2}$ receptors and $\alpha$-subunits of $\mathrm{G}_{\mathrm{oA}}$ and $\mathrm{G}_{\mathrm{i} 1}$ G-proteins were expressed in recombinant baculoviruses, which were constructed and generated according to Bac-to-Bac ${ }^{\circledR}$ Baculovirus Expression System manual (Life technologies) ${ }^{22}$.

$100 \mathrm{ml}$ of Sf9 cell suspension at a density of $2 \times 10^{6}$ cells $/ \mathrm{ml}$ were co-infected with baculoviruses encoding the $\mathrm{M}_{2}$ receptor and $\mathrm{G}_{\mathrm{oA}}$ or $\mathrm{G}_{\mathrm{i} 1}$ at a multiplicity of infection $\mathrm{MOI}=0.1: 0.1$. All infections were allowed to proceed for $69 \mathrm{~h}$. Infected cells were harvested by centrifugation at $500 \times \mathrm{g}$ for $5 \mathrm{~min}$ and frozen at $-80^{\circ} \mathrm{C}$.

The pellets of harvested cells were suspended in the ice-cold homogenization medium (100 mM $\mathrm{NaCl}, 20 \mathrm{mM}$ Na-HEPES, $10 \mathrm{mM}$ EDTA, pH =7.4) and homogenized on ice by two $30 \mathrm{sec}$ strokes using a Polytron homogenizer (Ultra-Turrax; Janke \& Kunkel GmbH \& Co. KG, IKALabortechnik, Staufen, Germany) with a 30-sec pause between strokes. Cell homogenates were centrifuged for $5 \mathrm{~min}$ at $1000 \times \mathrm{g}$. The supernatant was collected and centrifuged for $30 \mathrm{~min}$ at $30,000 \times$ g. Pellets were suspended in the washing medium $(100 \mathrm{mM} \mathrm{NaCl}, 10 \mathrm{mM} \mathrm{MgCl} 2,20$ $\mathrm{mM} \mathrm{Na}$-HEPES, $\mathrm{pH}=7.4$ ), left for $30 \mathrm{~min}$ at $4{ }^{\circ} \mathrm{C}$, and then centrifuged again for $30 \mathrm{~min}$ at $30,000 \times \mathrm{g}$. The resulting membrane pellets were kept at $-80^{\circ} \mathrm{C}$ until assayed.

\section{GTP $\gamma$ S binding}

Agonist stimulated $\left[{ }^{35} \mathrm{~S}\right] \mathrm{GTP} \gamma \mathrm{S}$ binding was performed as currently described ${ }^{23}$. Briefly, it was performed in 96 -well plates at $30^{\circ} \mathrm{C}$ in the incubation medium described above that was supplemented with freshly prepared dithiothreitol at a final concentration of $1 \mathrm{mM}$. Suspension of membranes of Sf9 cells expressing $\mathrm{M}_{2}+\mathrm{G}_{\mathrm{oA}}$ or $\mathrm{M}_{2}+\mathrm{G}_{\mathrm{i} 1}$ were preincubated with GDP and agonists for $15 \mathrm{~min}$, then $\left[{ }^{35} \mathrm{~S}\right] \mathrm{GTP} \gamma \mathrm{S}$ was added for an additional $20 \mathrm{~min}$. The final concentration of GDP and $\left[{ }^{35} \mathrm{~S}\right] \mathrm{GTP} \gamma \mathrm{S}$ was $20 \mu \mathrm{M}$ and $500 \mathrm{pM}$, respectively. Nonspecific binding was determined in the presence of $1 \mu \mathrm{M}$ non-labelled GTP $\gamma \mathrm{S}$. Incubations were terminated by filtration through GF/C filtration plates (Millipore) using a Brandel cell harvester (Perkin Elmer, 
USA). Plates were dried in microwave oven 800W 3 min and then $40 \mu 1$ of ROTISZINT® Eco

Plus (ROTH) was added. The plates were counted in the Wallac Microbeta scintillation counter.

\section{References}

1. Black, J. W. \& Leff, P. Operational models of pharmacological agonism. Proc. R. Soc. London. Ser. B, Biol. Sci. 220, 141-62 (1983).

2. Black, J. W., Leff, P., Shankley, N. P. \& Wood, J. An operational model of pharmacological agonism: the effect of $\mathrm{E} /[\mathrm{A}]$ curve shape on agonist dissociation constant estimation. Br. J. Pharmacol. 84, 561-71 (1985).

3. Gesztelyi, R. et al. The Hill equation and the origin of quantitative pharmacology. Arch. Hist. Exact Sci. 66, 427-438 (2012).

4. Jakubík, J. et al. Applications and limitations of fitting of the operational model to determine relative efficacies of agonists. Sci. Rep. 9, 4637 (2019).

5. Furchgott, R. F. The use of $\beta$-haloalkylamines in the diferentiation of receptors and in the determination of dissociation constants of receptor-agonist complexes. Adv. Drug Res. 3, 21-55 (1966).

6. Ehlert, F. J., Griffin, M. T., Sawyer, G. W. \& Bailon, R. A simple method for estimation of agonist activity at receptor subtypes: comparison of native and cloned M3 muscarinic receptors in guinea pig ileum and transfected cells. J. Pharmacol. Exp. Ther. 289, 98192 (1999).

7. Kenakin, T. \& Christopoulos, A. Signalling bias in new drug discovery: detection, quantification and therapeutic impact. Nat. Rev. Drug Discov. 12, 205-16 (2013).

8. Lefkowitz, R. J. A brief history of G-protein coupled receptors (Nobel Lecture). Angew. Chem. Int. Ed. Engl. 52, 6366-78 (2013).

9. Kenakin, T., Watson, C., Muniz-Medina, V., Christopoulos, A. \& Novick, S. A simple method for quantifying functional selectivity and agonist bias. ACS Chem. Neurosci. 3, 193-203 (2012).

10. Christopoulos, A. \& El-Fakahany, E. E. Qualitative and quantitative assessment of relative agonist efficacy. Biochem. Pharmacol. 58, 735-748 (1999).

11. Kenakin, T. P. Biased signalling and allosteric machines: New vistas and challenges for 
drug discovery. Br. J. Pharmacol. 165, 1659-1669 (2012).

12. Keov, P. et al. Molecular mechanisms of bitopic ligand engagement with the M1 muscarinic acetylcholine receptor. J. Biol. Chem. 289, 23817-37 (2014).

13. Luttrell, L. M., Maudsley, S. \& Bohn, L. M. Fulfilling the Promise of 'Biased' G ProteinCoupled Receptor Agonism. Mol. Pharmacol. 88, 579-88 (2015).

14. Stott, L. A., Hall, D. A. \& Holliday, N. D. Unravelling intrinsic efficacy and ligand bias at $\mathrm{G}$ protein coupled receptors: A practical guide to assessing functional data. Biochem. Pharmacol. 101, 1-12 (2016).

15. Burgueño, J. et al. A Complementary Scale of Biased Agonism for Agonists with Differing Maximal Responses. Sci. Rep. 7, 15389 (2017).

16. Kenakin, T. A Scale of Agonism and Allosteric Modulation for Assessment of Selectivity, Bias, and Receptor Mutation. Mol. Pharmacol. 92, 414-424 (2017).

17. Kenakin, T. P. Agonists: The Measurement of Affinity and Efficacy in Functional Assays. in A Pharmacology Primer 85-117 (Academic Press, 2014). doi:10.1016/b9780-12-407663-1.00005-3.

18. Gregory, K. J., Giraldo, J., Diao, J., Christopoulos, A. \& Leach, K. Evaluation of Operational Models of Agonism and Allosterism at Receptors with Multiple Orthosteric Binding Sites. Mol. Pharmacol. 97, 35-45 (2020).

19. Roche, D., van der Graaf, P. H. \& Giraldo, J. Have many estimates of efficacy and affinity been misled? Revisiting the operational model of agonism. Drug Discov. Today 21, 1735-1739 (2016).

20. Griffin, M. T., Figueroa, K. W., Liller, S. \& Ehlert, F. J. Estimation of agonist activity at $\mathrm{G}$ protein-coupled receptors: analysis of M2 muscarinic receptor signaling through Gi/o,Gs, and G15. J. Pharmacol. Exp. Ther. 321, 1193-207 (2007).

21. Jakubík, J., Randáková, A., Chetverikov, N., El-Fakahany, E. E. \& Doležal, V. The operational model of allosteric modulation of pharmacological agonism. Sci. Rep. 10, $14421(2020)$.

22. Anderson, D. et al. Rapid generation of recombinant baculovirus and expression of foreign genes using the BAC-to-BAC Baculovirus expression system. Focus (Madison). 
17, 53-58 (1995).

23. Randáková, A. et al. Agonist-Specific Conformations of the M2 Muscarinic Acetylcholine Receptor Assessed by Molecular Dynamics. J. Chem. Inf. Model. 60, 2325-2338 (2020).

\section{Acknowledgements}

This research was funded by the Czech Academy of Sciences institutional support [RVO:67985823] and the Grant Agency of the Czech Republic grant [19-05318S].

\section{Author contributions}

JJ derived equation for theoretical models and wrote python code for model data analysis. AR and DN designed, conducted experiments and analysed experimental data. All authors contributed to writing the manuscript, read and approved final version of the manuscript.

\section{Conflict of interest}

The authors declare no conflict of interest.

\section{Availability of data}

The data that support the findings of this study are available from the corresponding author upon reasonable request. 


\section{Supplementary Files}

This is a list of supplementary files associated with this preprint. Click to download.

- python.tar.gz 